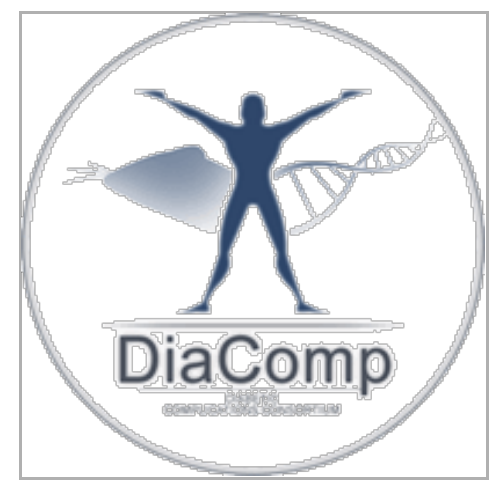

VERSION 2

SEP 27, 2019

\section{open $\boldsymbol{\gamma}$ Access}

DOI:

dx.doi.org/10.17504/protocol s.io. 7 rbhm $2 n$

\section{External link:}

https://www.diacomp.org/shar ed/document.aspx?

$\mathrm{id}=54 \&$ docType $=$ Protocol

Protocol Citation: Eva Feldman 2019. Neuropathy Phentoyping Protocols - Nerve Conduction Velocity. protocols.io

https://dx.doi.org/10.17504/p rotocols.io. 7 rbhm $2 \mathrm{n}$

\section{MANUSCRIPT CITATION:}

Eid SA, Feldman EL, Advances in diet-induced rodent models of metabolically acquired peripheral neuropathy. Disease Models \& Mechanisms 14(11). doi: 10.1242/dmm.049337

License: This is an open access protocol distributed under the terms of the Creative Commons Attribution License, which permits unrestricted use, distribution, and reproduction in any medium, provided the original author and source are credited

\section{(3) Neuropathy Phentoyping Protocols - Nerve Conduction Velocity V.2}

Eva Feldman ${ }^{1}$

${ }^{1}$ University of Michigan - Ann Arbor

Diabetic Complications Consortium

Tech. support email: rmcindoe@augusta.edu

Lili Liang

\section{ABSTRACT}

\section{Summary:}

\section{Phenotyping of Rodents for the Presence of Diabetic Neuropathy}

In man, the development of diabetic neuropathy is dependent on both the degree of glycemic control and the duration of diabetes. Diabetic neuropathy is a progressive disorder, with signs and symptoms that parallel the loss of nerve fibers over time. Consequently, assessments of neuropathy in mice are not performed at one time point, but are characterized at multiple time points during a 6 month period of diabetes. The degree of diabetes is evaluated in 2 ways: tail blood glucose measured following a 6 hour fast and glycated hemoglobin levels. The initial degree of neuropathy is screened using the methods discussed below. Detailed measures of neuropathy are employed when the initial screening instruments indicate a profound or unique phenotypic difference. This document contains protocols used by the DiaComp staff to examine and measure diabetic neuropathy at the whole animal, tissue and cellular levels.

\section{Diabetic Complication:}

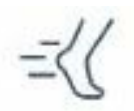

Neuropathy 
Protocol status: Working We use this protocol and it's working

Created: Sep 27, 2019

Last Modified: Sep 27, 2019

\section{PROTOCOL integer ID:} 28163

Keywords: Neuropathy Phentoyping, Diabetic Neuropathy, Nerve Conduction Velocity

\section{MATERIALS}

\section{Equipment:}

- Nicolet VikingQuest Portable System with Nerve Conduction Studies VikingQuest software run on Windows NT.

- HP laser printer.

- Nicolet $12 \mathrm{~mm} .4 \mathrm{~mm}$ diameter disposable platinum EEG subdermal needles.

- Nicolet disposable ground.

- Heating lamp.

Heating pad.

- Flexible tape measure.

- 8"x 8" Styrofoam .

- Ketastet 100mg/ml (3 parts) and Rompun 20 mg/ml (1 part) --- stock solution should be diluted $1 / 10$ for mice and as is for rats.

\section{Settings:}

1 Motor tests:

- Duration $.02 \mathrm{~ms}$.

- Range $25 \mathrm{Ma}$.

- Low frequency filter $1 \mathrm{~Hz}$.

- High frequency filter $10 \mathrm{kHz}$.

- Sensitivity $1 \mathrm{mV}$.

- Time 2 ms/div.

\section{Sensory test:}

- Duration .02 ms.

- Range $25 \mathrm{~mA}$.

- Low frequency filter $1 \mathrm{~Hz}$.

- High frequency filter $10 \mathrm{kHz}$.

- Sensitivity $50 \mu \mathrm{V}$.

- Time $2 \mathrm{~ms} /$ div.

\section{Procedure:}

2 - To confirm the presence of diabetic neuropathy, nerve conduction velocity (NCV) studies will be performed. The animals will be anesthetized with $30 / 2.5 \mathrm{mg} / \mathrm{kg}$ ketamine/xylazine to prevent discomfort. Body temperature will be monitored with a dermal temperature probe and maintained at $32^{\circ} \mathrm{C}$ with a warming lamp during NCV. Body temperature will be maintained at $37^{\circ} \mathrm{C}$ after NCV using a warming pad to ease animal stress from anesthetic. The nerve studies will last less than 30 min per rat or mouse. The electrodes will be cleaned with $70 \%$ alcohol between animals to maintain pathogen-free status. 
- Sciatic-tibial motor NCV will be determined by stimulating distally at the sciatic notch and distally at the knee via bipolar electrodes with supramaximal stimulation. The Sciatic-tibial motor NCV is calculated using two points of stimulation along the nerve and measuring the resultant latency. Latency is measured from initial onset to maximum negative peak.

- Tail motor latency will be determined by stimulating distally along the tail at a recorded distance of $3 \mathrm{~cm}$. Latency is measured from initial onset to maximum negative peak.

- Tail sensory NCV will be determined by stimulating proximally along the tail at a recorded distance of $3 \mathrm{~cm}$. Latency is measured from initial onset to maximum negative peak.

\section{$3 \quad$ Figure 4}

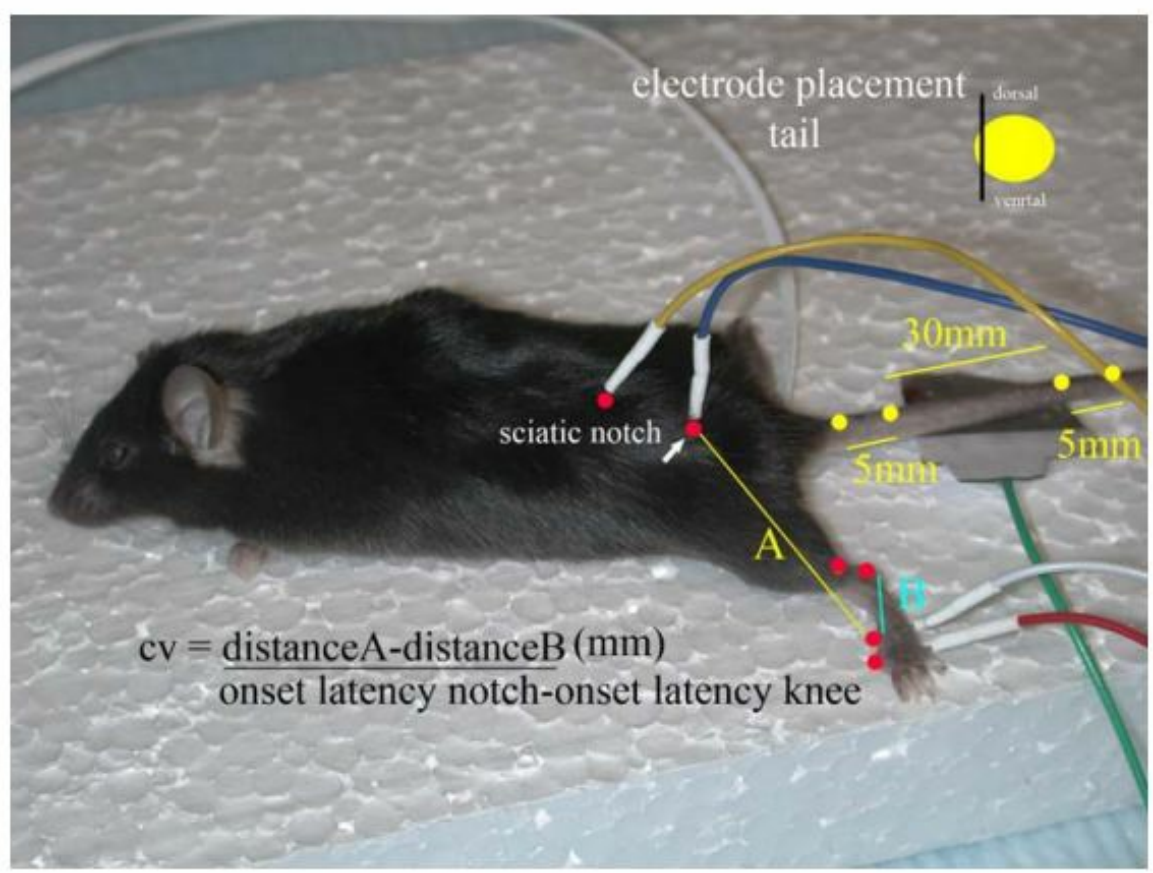




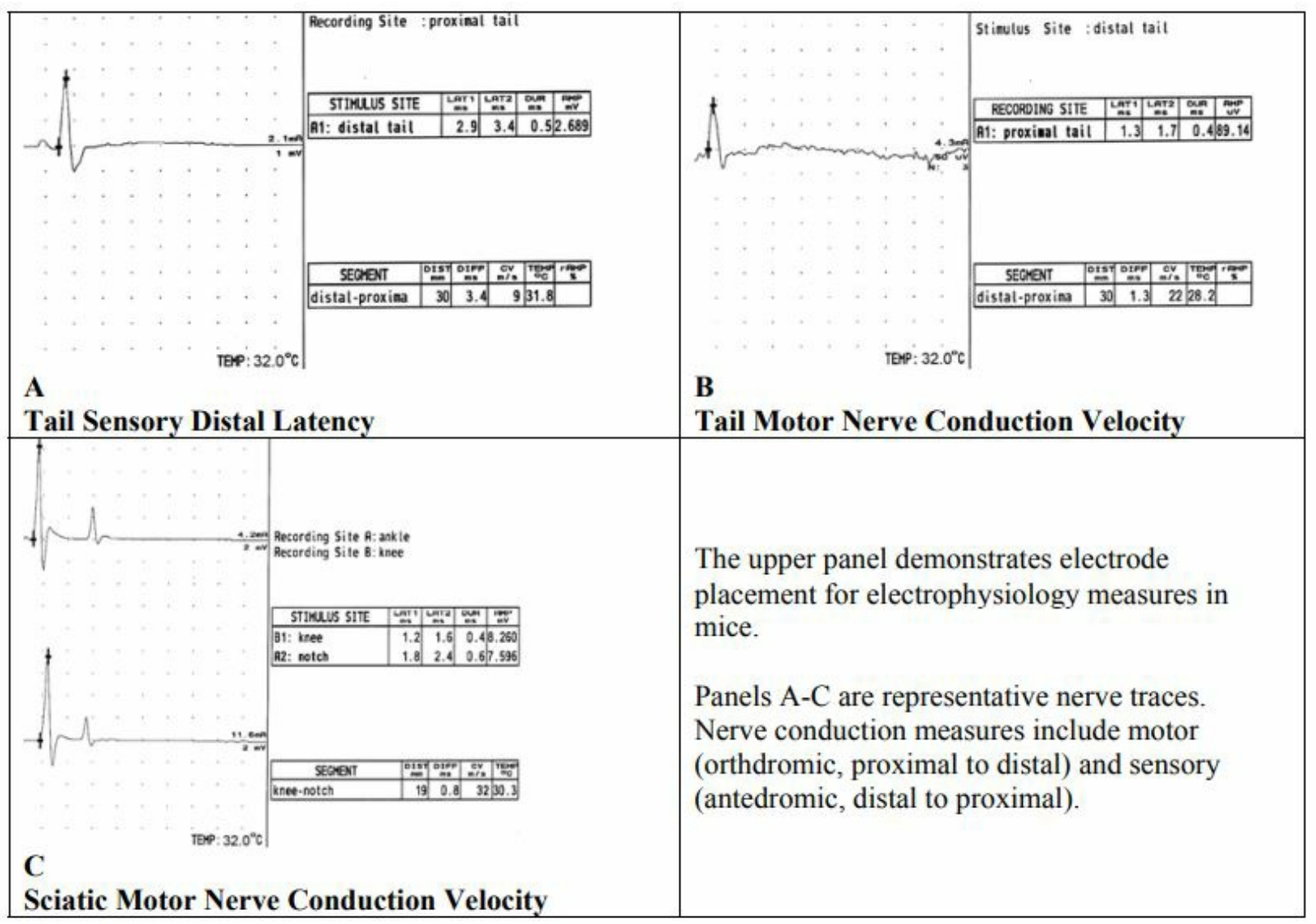

